\title{
Parallel Interference Cancellation for Uplink Multirate Overlay CDMA Channels
}

\author{
Hongbo Yan, Member, IEEE, and Sumit Roy, Senior Member, IEEE
}

\begin{abstract}
To provide new and/or higher rate wireless services with limited spectrum resources, frequency overlay has been naturally proposed to accommodate the new and legacy systems in a common band. In this paper, we address the multiuser detection problem for overlaid code-division multiple-access (CDMA) scenarios. However, in contrast to the well-studied conventional single-rate CDMA, miscellaneous systems overlay almost always indicates the presence of multirate traffic that introduces an additional degree of freedom in receiver design-i.e., differences in the symbol rates. In this paper, we concentrate on receiver design for multirate traffic, while assuming a lack of information exchange between the constituent (new and legacy) systems, as is commonplace in practice. We propose a receiver architecture based on linear parallel interference cancellation where the out-of-rate intersystem interference is estimated and subtracted by means of its characteristic subspace, thereby avoiding the need for the exact knowledge of signature waveforms of the interfering system. Simulation results validate our solution and show that the proposed receiver has better performance in various aspects than several other solutions for the same purpose.
\end{abstract}

Index Terms-Code-division multiple-access (CDMA), group-blind detection, multirate processing, parallel interference cancellation (PIC), spectral overlay.

\section{INTRODUCTION}

A S DEMANDS for personal wireless multimedia services continue to grow, the need for deployment of newer systems with larger bandwidth to support such applications becomes more acute. However, due to limitation of available bandwidth, frequency overlay of newer (data) services upon legacy (voice-oriented) systems is a likely solution. Naturally, code-division multiple access (CDMA) is the usual platform for such overlay, due to its multiple accessing capabilities and immunity to narrowband interference (NBI) [1], [2]. Simulation results in [3] showed that net system capacity can be considerably increased when a wideband CDMA (W-CDMA) system is laid upon the global system for mobile communications (GSM) network, which was further justified experimentally in [4] and [5]. The legacy GSM signal acts as a NBI to the wideband CDMA; the suppression of such signals (i.e., tone jammers or digitally modulated signals) has been well studied, see the excellent overview in [7] and references therein.

Paper approved by D. I. Kim, the Editor for Spread Spectrum Transmission and Access of the IEEE Communications Society. Manuscript received October 28, 2002; revised September 10, 2003 and March 26, 2004.

H. Yan is with Qualcomm Inc., San Diego, CA 92121 USA (e-mail: yanh@qualcomm.com).

S. Roy is with the Department of Electrical Engineering, University of Washington, Seattle, WA 98195 USA (e-mail: roy @ee.washington.edu).

Digital Object Identifier 10.1109/TCOMM.2004.838729
In this paper, we consider a canonical overlay scenario of wideband CDMA upon other existing narrowband CDMA (N-CDMA) systems. In contrast to mere NBI suppression for the wider-band system, joint receiver-design issues for such heterogeneous CDMA systems have hardly been addressed and constitute the focus of this paper.

Although our methods apply to situations involving multiple CDMA systems, for the sake of simplicity, we attend largely to the overlay case of two systems, termed N-CDMA and W-CDMA, respectively, in accordance to their relative signal bandwidth.

The difference in bandwidth of the constituent systems naturally leads to a multirate signal-processing formulation of this $\mathrm{N}-\mathrm{CDMA} / \mathrm{W}-\mathrm{CDMA}$ overlay. The received signal is processed over an interval which is the least common multiple of all the symbol periods, as in [13], to ensure wide-sense stationarity. Despite increased numerical complexity due to a larger observation vector size, the receiver design problem devolves into the familiar single-rate framework, but with equivalent multiple "fictitious" users corresponding to a physical user. The signature waveforms of the fictitious users in the interfering system are unknown at the receiver, due to the lack of coordination between different networks. Although the minimum mean-square error (MMSE) receiver developed in [12] needs only the signature waveform of the desired user, improved performance is naturally expected if knowledge of all known signature waveforms of users in the system of interest are exploited. In this paper, we therefore adopt a group-blind multiuser detection (MUD) perspective [14].

Parallel interference cancellation (PIC) for CDMA systems has attracted much interest lately, due to its structured architecture that facilitates easier implementation. Pioneered by [8], such multistage methods attempt to cancel multiple-access interference (MAI) based on the tentative decisions obtained at the previous stage. When hard decisions are used at the intermediate stages, as in [8], signal amplitudes need to be estimated to generate MAI estimates. Also, if a prior stage's decision for some user is erroneous, subtracting the corresponding MAI estimate will result in the familiar "error propagation" phenomena that contributes to progressively enhanced interference. To alleviate these problems, linear PIC schemes [9]-[11] were proposed which, until the final stage, exploit soft decisions that provide estimates for each user's information bit and amplitude. It is shown in [11] that linear PIC converges to the decorrelating detector as the number of stages $M \rightarrow \infty$, if the spectral radius of signature waveform cross-correlation matrix is less than two. In this paper, we intend to generalize this idea to the $\mathrm{N}-\mathrm{CDMA} / \mathrm{W}-\mathrm{CDMA}$ overlay scenario. While intrasystem MAI 


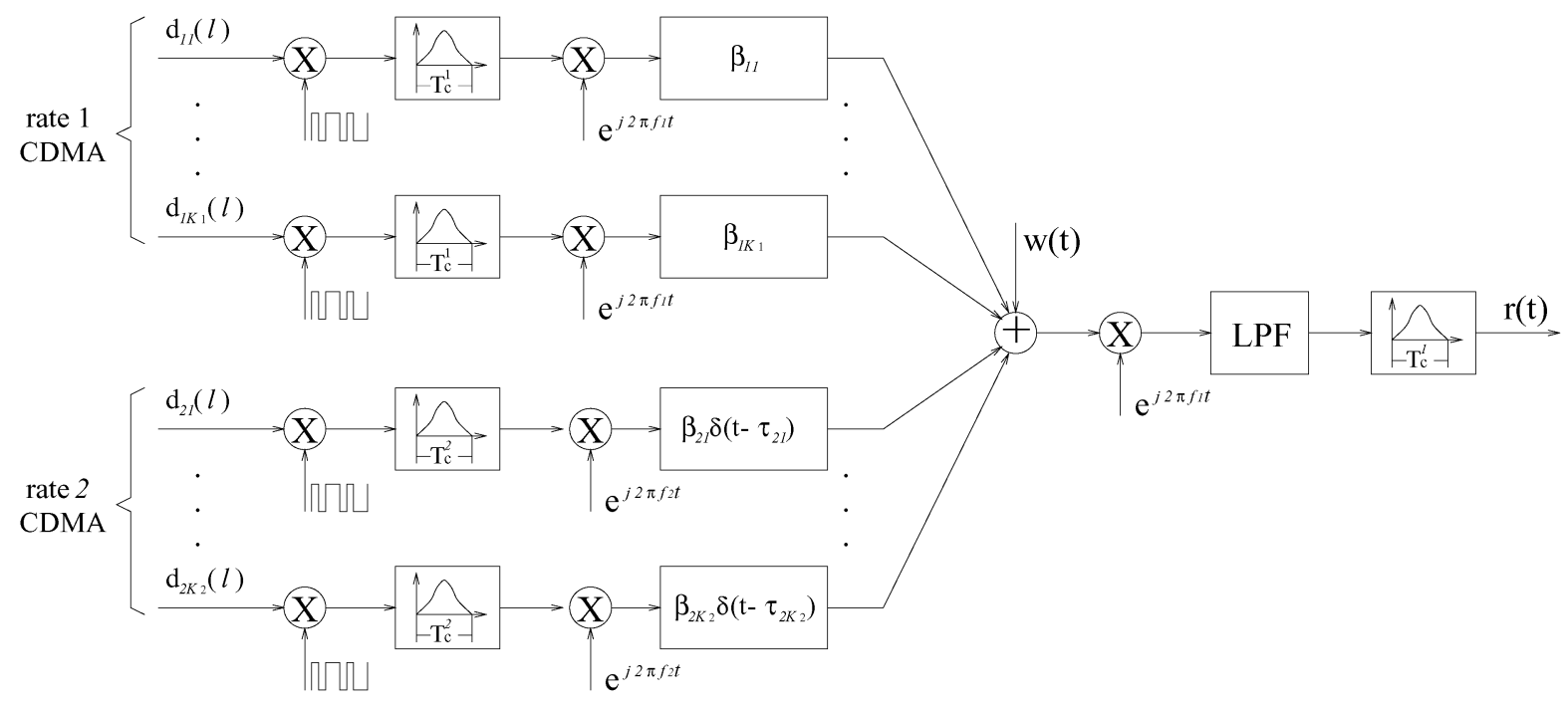

Fig. 1. Two-CDMA overlay.

can be estimated with the help of tentative decisions obtained with traditional matched filtering, out-of-rate intersystem MAI needs special attention, since the corresponding signature waveforms are unknown. Nevertheless, for out-of-rate MAI suppression, one only needs to estimate the subspace spanned by the unknown signature waveforms, but not the signature waveforms themselves.

Several group-blind multiuser CDMA detectors have been proposed in [14] which can easily be applied to our model. Note that the subspace to be estimated in [14] is spanned only by the out-of-rate interference components that are orthogonal to the known signature waveforms. In situations where known and unknown signature waveforms are highly correlated (such as due to the presence of a multipath ISI channel), the performance of such methods will degrade due to poor estimation of the number of interfering sources and their spanning subspace. We propose a robust subspace estimate by considering the whole out-of-rate interference. Finally, note that the proposed methods cannot be used for group-blind MUD in single-rate systems as in [14], as it specifically exploits the multirate received-signal structure for parameter estimation.

The rest of the paper is organized as follows. Section II describes the system model, Section III elaborates algorithm development, Section IV gives performance analysis, Section V presents the simulation results, and finally, Section VI concludes the paper.

\section{SYSTEM MODEL}

A generic system formulation is proposed, which translates multirate overlay scenarios into an equivalent single-rate system by introducing the concept of "fictitious" CDMA users. The model accommodates asynchronism, carrier offset of the out-ofrate system, and applies to a variety of applications. We assume that the rate-1 system in Fig. 1 is the one of interest and illustrate how variable processing gain (VPG) and variable chipping rate (VCR) multirate CDMA systems can also be included in this formulation.

\section{A. N-CDMA/W-CDMA Overlay}

For the N-CDMA/W-CDMA overlay system depicted in Fig. 1, the uplink received signal at the CDMA base station can be characterized by

$$
r(t)=r_{1}(t)+r_{2}(t)+w(t)
$$

where $r_{1}(t)$ is the signal component due to the desired CDMA system (either N-CDMA or W-CDMA), $r_{2}(t)$ is the interference from the other system, and $w(t)$ is the additive white Gaussian noise (AWGN) of variance $\sigma^{2}$. We assume that the three are mutually independent. $r_{i}(t)(i=1,2)$ is given by

$$
r_{i}(t)=\sum_{j=1}^{K_{i}} \sum_{l=-\infty}^{\infty} \beta_{i j} d_{i j}(l) s_{i j}\left(t-l T_{i}-\tau_{i j}\right) e^{j 2 \pi f_{i}\left(t-\tau_{i j}\right)}
$$

where $K_{i}$ is the number of active users in the $i$ th system operating at symbol rate $1 / T_{i},\left\{d_{i j}(l)\right\}$ 's are zero-mean mutually independent independent and identically distributed (i.i.d.) information sequences of normalized unit variance, $s_{i j}(t)$ is the signature waveform of unit energy, $f_{i}$ is the system's carrier frequency, $\beta_{i j}$ is the signal amplitude, and $\tau_{i j}$ is the propagation delay uniformly distributed within $\left[0 T_{i}\right]$. The signature waveform $s_{i j}(t)$ is confined to $\left[0 T_{i}\right]$ and defined as

$$
s_{i j}(t)=\sum_{k=0}^{L_{i}-1} c_{i j}(k) g_{i}\left(t-k T_{c}^{i}\right)
$$

where $c_{i j}(t)$ is user $(i j)$ 's spreading code, $g_{i}(t)$ is the chip pulse, $T_{c}^{i}$ and $L_{i}$ are the respective chip duration and processing gain with $T_{c}^{i}=T_{i} / L_{i}$. Note that in contrast to $\tau_{1 j}$ and $s_{1 j}(t), \tau_{2 j}$ and $s_{2 j}(t)$ are generally unknown, due to the lack of coordination between the two systems. In the following, we will assume the same processing gain for both systems, i.e., $L=L_{1}=L_{2}$, thus symbol rate is proportional to bandwidth occupancy. While untrue in practice, the resulting simplicity leads to some insights that would not be otherwise available from an analytical study; at any rate, the model can be easily generalized to unequal processing gain scenarios. Based on this assumption, the system with the highest symbol rate (and hence, chip rate) is identified as the W-CDMA system, and the other is the overlaid 
N-CDMA system. After receiver front-end carrier demodulation and low-pass filtering, $r(t)$ can be rewritten as

$$
\begin{aligned}
& r(t)=\sum_{i=1}^{2} \sum_{j=1}^{K_{i}} \sum_{l=-\infty}^{\infty} \beta_{i j} d_{i j}(l) s_{i j}(\left.t-l T_{i}-\tau_{i j}\right) \\
& \times e^{j 2 \pi \Delta f_{i}\left(t-\tau_{i j}\right)}+w(t)
\end{aligned}
$$

where $\Delta f_{1}=0$ and $\Delta f_{2}=f_{2}-f_{1}$ is the relative carrier offset of $f_{2}$ with respect to $f_{1}$.

Suppose the symbol-rate ratio is given by

$$
\frac{T_{1}}{p_{1}}=\frac{T_{2}}{p_{2}}
$$

where $p_{1}$ and $p_{2}$ are co-prime integers. Denote $P=p_{1} p_{2}, q_{i}=$ $P / p_{i}, T=q_{i} T_{i}$ and introduce

$$
d_{i j}^{(k)}(l)=d_{i j}\left(l q_{i}+k\right) e^{j 2 \pi\left(l q_{i}+k\right) \Delta f_{i} T_{i}} .
$$

Note that if we treat $d_{i j}^{(k)(l)}$, s as symbol streams from individual fictitious users and define the corresponding waveforms

$s_{i j}^{(k)}(t)= \begin{cases}s_{i j}\left(t-k T_{i}\right) e^{j 2 \pi \Delta f_{i}\left(t-k T_{i}\right)}, & t \in\left[k T_{i},(k+1) T_{i}\right] \\ 0, & \text { elsewhere }\end{cases}$

(4) can be rewritten as

$$
r(t)=\sum_{i=1}^{2} \sum_{j=1}^{K_{i}} \sum_{k=0}^{q_{i}} \sum_{l=-\infty}^{\infty} \beta_{i j} d_{i j}^{(k)}(l) s_{i j}^{(k)}\left(t-l T-\tau_{i j}\right)+w(t)
$$

indicating an equivalent single-rate (1/T) CDMA model of $\left(q_{1}+1\right) K_{1}+\left(q_{2}+1\right) K_{2}$ users. The corresponding signature waveforms $\left(s_{i j}^{(k)}(t)\right)$ are sketched in Fig. 2 for a single physical user in each system, where we have assumed W-CDMA is the desired system. For illustrative purposes, we also adopt the rectangular pulse shaping, and let $\Delta f_{2}=0$ for convenience, with the understanding that in real systems, the signature from waveforms of users from the interfering system are modulated by a residual carrier. Observe that the fictitious users derived from the same physical user are mutually orthogonal, since the nonzero parts of their signatures do not overlap.

Sampling the received signal at interval $\delta=T_{c}^{i} / p_{i}$ and collecting data for an observation interval $T$ yield the discrete-time model

$$
\mathbf{r}(n)=\mathbf{S A d}(n)+\mathbf{w}(n)
$$

where

$$
\begin{aligned}
\mathbf{r}(n) & =[r(n L P \delta) \ldots r((n L P+L P-1) \delta)]^{T} \\
\mathbf{w}(n) & =[w(n L P \delta) \ldots w((n L P+L P-1) \delta)]^{T} \\
\mathbf{A} & =\left[\begin{array}{ll}
\mathbf{A}_{1} & \\
& \mathbf{A}_{2}
\end{array}\right] \\
\mathbf{S} & =\left[\begin{array}{ll}
\mathbf{S}_{1} & \mathbf{S}_{2}
\end{array}\right] \\
\mathbf{d}(n) & =\left[\begin{array}{ll}
\mathbf{d}_{1}^{T}(n) & \mathbf{d}_{2}^{T}(n)
\end{array}\right]^{T}
\end{aligned}
$$

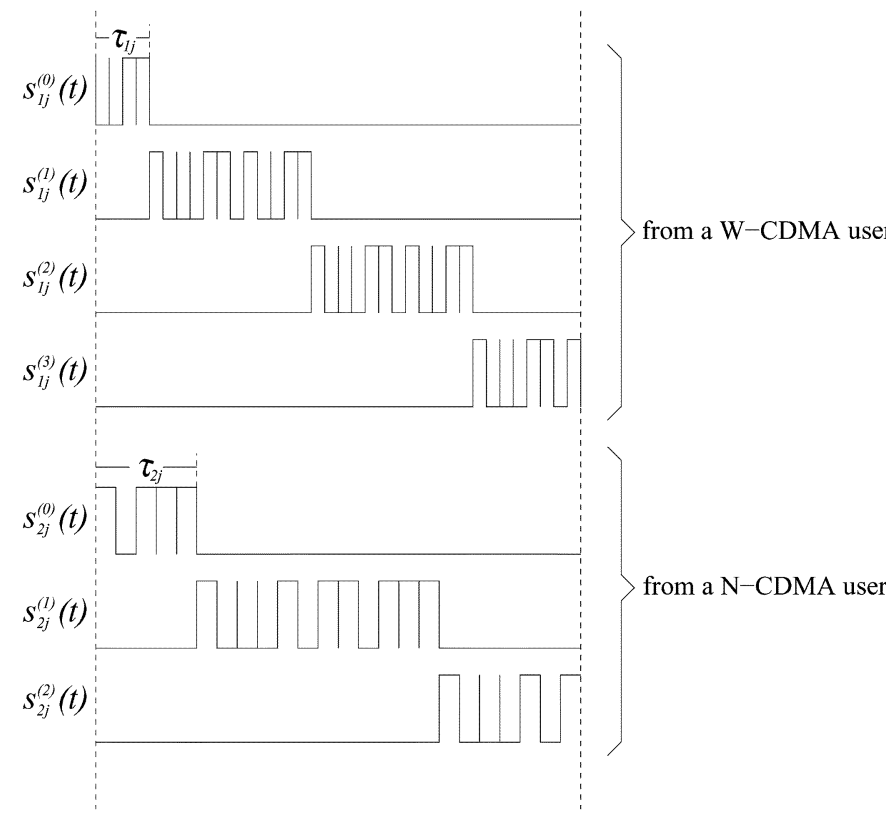

Fig. 2. Fictitious signature waveforms.

with

$$
\begin{aligned}
\mathbf{A}_{i}= & \operatorname{diag}(\underbrace{\beta_{i 1} \ldots \beta_{i 1}}_{q_{i}+1} \ldots \underbrace{\beta_{i K_{i}} \ldots \beta_{i K_{i}}}_{q_{i}+1}) \\
\mathbf{S}_{i}= & {\left[\mathbf{s}_{i 1}^{(-1)} \mathbf{s}_{i 1}^{(0)} \ldots \mathbf{s}_{i 1}^{\left(q_{i}-1\right)} \ldots \mathbf{s}_{i K_{i}}^{(-1)} \mathbf{s}_{i K_{i}}^{(0)} \ldots \mathbf{s}_{i K_{i}}^{\left(q_{i}-1\right)}\right] } \\
\mathbf{d}_{i}(n)= & {\left[d_{i 1}^{\left(q_{i}-1\right)}(n-1) d_{i 1}^{(0)}(n) \ldots d_{i 1}^{\left(q_{i}-1\right)}(n) \ldots\right.} \\
& \left.d_{i K_{i}}^{\left(q_{i}-1\right)}(n-1) d_{i K_{i}}^{(0)}(n) \ldots d_{i K_{i}}^{\left(q_{i}-1\right)}(n)\right]^{T} .
\end{aligned}
$$

Let $m_{i j}=\left\lceil\tau_{i j} / \delta\right\rceil$, where $\lceil x\rceil$ is the smallest integer no less than $x, \gamma_{i j}=m_{i j} \delta-\tau_{i j}$, and $s_{i j}(l)=s_{i j}(l \delta+$ $\left.\gamma_{i j}\right) e^{j 2 \pi \Delta f_{i}\left(l \delta+\gamma_{i j}\right)}\left(0 \leq l \leq L p_{i}-1\right)$, the vector elements of $\mathbf{S}_{i}$ are defined as shown in (10) at the bottom of the next page. The dimensions of the listed vectors and matrices can thus be determined

$$
\begin{aligned}
& \mathbf{r}(n): L P \times 1 \\
& \mathbf{w}(n): L P \times 1 \\
& \mathbf{A}:\left(\left(q_{1}+1\right) K_{1}+\left(q_{2}+1\right) K_{2}\right)\left(\left(q_{1}+1\right) K_{1}+\left(q_{2}+1\right) K_{2}\right) \\
& \mathbf{S}: L P \times\left(\left(q_{1}+1\right) K_{1}+\left(q_{2}+1\right) K_{2}\right) \\
& \mathbf{d}(n):\left(\left(q_{1}+1\right) K_{1}+\left(q_{2}+1\right) K_{2}\right) \times 1 .
\end{aligned}
$$

Now (9) characterizes a single-rate CDMA system of processing gain $L P$ and $\left(q_{1}+1\right) K_{1}+\left(q_{2}+1\right) K_{2}$ users whose signature waveforms are the column vectors of $\mathbf{S}$. In the following, we will drop the time index $n$ in (9) for simplicity wherever feasible.

\section{B. VPG/VCR Multirate CDMA Systems}

VPG/VCR multirate CDMA systems have been proposed for third-generation $(3 \mathrm{G})$ cellular standards. In VCR CDMA systems, all the users have the same processing gain, but possibly different symbol rates. This coincides with the above-described 
N-CDMA/W-CDMA overlay situation. In the literature, it is usually assumed that all the users share the same carrier, thus the model (9) can accommodate VCR CDMA systems as a special case where $\Delta f_{i}=0$ for all $i$. In VPG CDMA systems, all the users share the same bandwidth (equivalently, the same chip rate $1 / T_{c}$ ), and therefore, the processing gains are proportional to symbol rates. Suppose

$$
\frac{T_{1}}{L_{1}}=\frac{T_{2}}{L_{2}}=T_{c}
$$

where $L_{i}$ is the processing gain of rate $i$ users. Sampling $r(t)$ with interval $\delta=T_{i} / p L_{i}$, where $p$ is some oversampling factor with respect to the chip rate, yields exactly the same model as (9). For both VPG and VCR CDMA systems, $\tau_{i, j}$ 's are assumed known for any $i, j$. Now the dimensions of $\mathbf{A}, \mathbf{S}$, and $\mathbf{b}$ are, respectively, $\sum_{i=1}^{2}\left(q_{i}+1\right) K_{i} \times \sum_{i=1}^{2}\left(q_{i}+1\right) K_{i}, p\left(q_{1}+1\right) L_{1} \times$ $\sum_{i=1}^{2}\left(q_{i}+1\right) K_{i}$, and $\sum_{i=1}^{2}\left(q_{i}+1\right) K_{i} \times 1$. Note that while complete knowledge of the signature waveform matrix $\mathbf{S}$ is available in the VPG and VCR CDMA systems, only a submatrix of $\mathbf{S}$ is available in the overlay application.

\section{THE PIC RECEIVERS}

As mentioned before, to apply PIC to overlay communications, we need to estimate out-of-rate interference $\mathbf{r}_{2}=\mathbf{S}_{2} \mathbf{A}_{2} \mathbf{d}_{2}$ without the knowledge of $\mathbf{S}_{2}$. Denote $\mathbf{B}_{I}$ as an orthonormal basis of the interfering signature waveform matrix $\mathbf{S}_{2}$, then it follows that

$$
\mathbf{S}_{2}=\mathbf{B}_{I} \Gamma
$$

for some $\Gamma$. It is easy to see that

$$
\begin{aligned}
\mathbf{B}_{I} \mathbf{B}_{I}^{H} \mathbf{r}_{2} & =\mathbf{B}_{I} \mathbf{B}_{I}^{H} \mathbf{S}_{2} \mathbf{A}_{2} \mathbf{d}_{2}=\mathbf{B}_{I} \mathbf{B}_{I}^{H} \mathbf{B}_{I} \Gamma \mathbf{A}_{2} \mathbf{d}_{2} \\
& =\mathbf{B}_{I} \Gamma \mathbf{A}_{2} \mathbf{d}_{2}=\mathbf{r}_{2}
\end{aligned}
$$

i.e., the projection of out-of-rate interference $\mathbf{r}_{2}$ onto $\mathbf{B}_{I}$ is itself. The observation indicates that $\mathbf{r}_{2}$ can be extracted from $\mathbf{r}$, provided that an orthonormal basis of $\mathbf{S}_{2}$ is obtained. Thus assuming $\mathbf{B}_{I}$ is available, the receiver can be constructed as in Fig. 3 [9]: $\mathbf{S}_{1 j}=\left[\mathbf{s}_{1 j}^{(-1)} \mathbf{s}_{1 j}^{(0)} \ldots \mathbf{s}_{1 j}^{\left(q_{1}-1\right)}\right]$ is the matrix of a fictitious signature waveform derived from the $j$ th physical user of

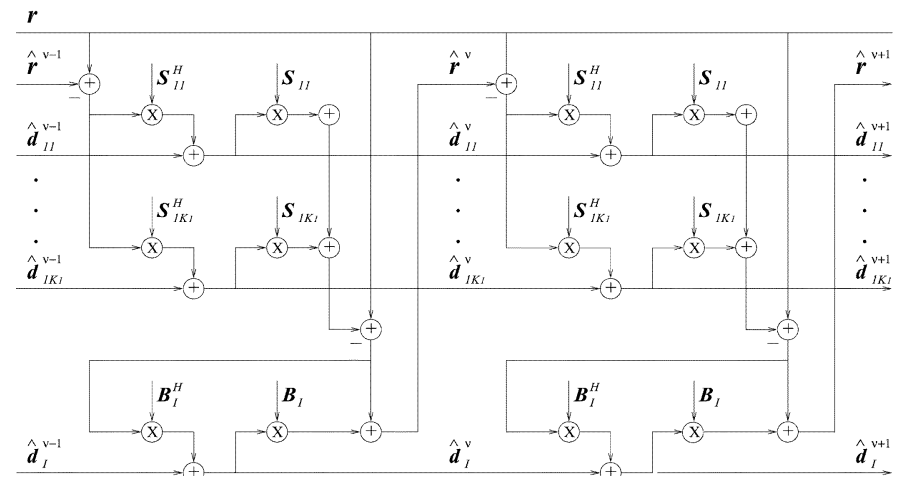

Fig. 3. Stage $\nu$ and $\nu+1$ of the linear PIC receiver. $\hat{\mathbf{d}}_{1 j}^{0}=\mathbf{0}, \hat{\mathbf{d}}_{I}^{0}=\mathbf{0}$.

the desired system $\hat{d}_{1, j}^{\nu}=\left[\hat{d}_{1, j}^{(q-1)}(n-1) \hat{d}_{1, j}^{(0)}(n) \hat{d}_{1, j}^{(q-1)}(n)\right]^{T}$ is the estimate at stage $\nu$ for the $j$ th physical user's amplitude/symbol within the block. Note that these symbols do not interfere with each other, due to the orthogonality between the fictitious signature waveforms. $\hat{\mathbf{d}}_{I}^{\nu}$ is the estimate of $\mathbf{d}_{I}=\mathbf{B}_{I}^{H} \mathbf{r}_{2}$ at stage $\nu$. The decision statistics at stage $\nu+1$ for user $j$ is given by

$$
\begin{aligned}
\hat{\mathbf{d}}_{1 j}^{\nu+1} & =\mathbf{S}_{1 j}^{H}\left(\mathbf{r}-\sum_{\substack{k=1 \\
k \neq j}}^{K_{1}} \mathbf{S}_{1 k} \hat{\mathbf{d}}_{1 k}^{\nu}-\mathbf{B}_{I} \hat{\mathbf{d}}_{I}^{\nu}\right) \\
& =\mathbf{S}_{1 j}^{H}\left(\mathbf{r}-\sum_{k=1}^{K_{1}} \mathbf{S}_{1 k} \hat{\mathbf{d}}_{1 k}^{\nu}-\mathbf{B}_{I} \hat{\mathbf{d}}_{I}^{\nu}\right)+\hat{\mathbf{d}}_{1 j}^{\nu} \\
\hat{\mathbf{d}}_{I}^{\nu+1} & =\mathbf{B}_{I}^{H}\left(\mathbf{r}-\sum_{k=1}^{K_{1}} \mathbf{S}_{1 k} \hat{\mathbf{d}}_{1 k}^{\nu+1}\right) .
\end{aligned}
$$

Note that the structure is different from the conventional PIC receiver in the sense that symbol estimates at the current stage are used for interference estimation. Such a strategy is chosen because exact knowledge of $\mathbf{S}_{1}$ tends to generate more reliable results than the estimated $\mathbf{B}_{I}$. Letting $\mathcal{S}=\left[\begin{array}{ll}\mathbf{S}_{1} & \mathbf{B}_{I}\end{array}\right], \hat{\mathbf{d}}_{1}=$ $\left[\hat{\mathbf{d}}_{11}^{T} \ldots \hat{\mathbf{d}}_{1 K_{1}}^{T}\right]^{T}$ and $\hat{\mathbf{d}}=\left[\hat{\mathbf{d}}_{1}^{T} \hat{\mathbf{d}}_{I}^{T}\right]^{T}$, the decision rule is given by

$$
\hat{\mathbf{d}}_{1}^{\nu+1}=\mathbf{S}_{1}^{H} \mathbf{r}+\left(\mathbf{I}-\mathbf{S}_{1}^{H} \mathcal{S}\right) \hat{\mathbf{d}}^{\nu} .
$$

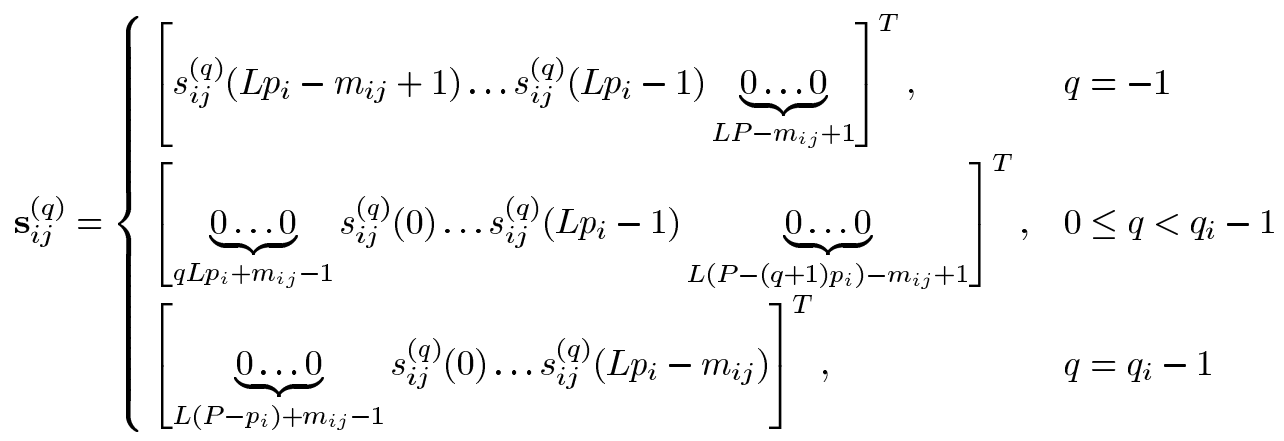


Denote by $\mathbf{F}_{M}$ the equivalent filter of an $M$-stage PIC receiver, i.e., $\hat{\mathbf{d}}_{1}^{M}=\mathbf{F}_{M}^{H} \mathbf{r}$. Together with initial condition $\hat{\mathbf{d}}^{0}=\mathbf{0}$, the above recursion yields

$$
\mathbf{F}_{M}^{H}=\sum_{\nu=0}^{M}\left(\mathbf{I}-\mathbf{S}_{1}^{H} \mathcal{S}\right)^{\nu} \mathbf{S}_{1}^{H} .
$$

In the following, we concentrate on the estimation of $\mathbf{B}_{I}$ when the system of interest (rate 1) is, respectively, W-CDMA or N-CDMA.

\section{A. W-CDMA as the Desired System}

The autocorrelation matrix of $\mathbf{r}$ is given by

$$
\begin{aligned}
\mathbf{R} & =E\left(\mathbf{r r}^{H}\right)=\mathbf{R}_{1}+\mathbf{R}_{2}+\sigma^{2} \mathbf{I} \\
& =\mathbf{S}_{1} \mathcal{A}_{1} \mathbf{S}_{1}^{H}+\mathbf{S}_{2} \mathcal{A}_{2} \mathbf{S}_{2}^{H}+\sigma^{2} \mathbf{I}
\end{aligned}
$$

where $\mathbf{R}_{1}=E\left(\mathbf{r}_{1} \mathbf{r}_{1}^{H}\right), \mathbf{R}_{2}=E\left(\mathbf{r}_{2} \mathbf{r}_{2}^{H}\right), \mathcal{A}_{1}=\mathbf{A}_{1} \mathbf{A}_{1}^{H}$, and $\mathcal{A}_{2}=\mathbf{A}_{2} \mathbf{A}_{2}^{H}$. Clearly, $\mathbf{B}_{I}$ can be obtained by means of eigenvalue decomposition on $\mathbf{R}_{2}$. Toward that end, $\mathbf{R}_{1}$ must be subtracted from $\mathbf{R}$. This necessitates estimation of the desired signals' magnitude matrix $\mathcal{A}_{1}$. For traditional single-rate CDMA systems, this problem has been addressed in [16]. We thus extract the desired signal component with a Wiener filter, which is given by

$$
H(f)=\frac{\gamma_{1} p_{2} \mathcal{Z}_{W}(f)}{\gamma_{1} p_{2} \mathcal{Z}_{W}(f)+\gamma_{2} p_{1} \mathcal{Z}_{N}(f)+\sigma^{2}}
$$

where $\gamma_{i}=\sum_{j=1}^{K_{i}} \beta_{i j}^{2}(i=1,2), \mathcal{Z}_{W}(f)$, and $\mathcal{Z}_{N}(f)$ are the respective power spectrum density (PSD) functions of the W-CDMA and N-CDMA users. Note that the dilemma is that we need signal amplitudes to design the filter. To get around this problem, we modify (19) to

$$
H(f)=\frac{\mathcal{Z}_{W}(f)}{\mathcal{Z}_{W}(f)+\mathcal{Z}_{N}(f)}
$$

which is called a modified Wiener filter. We use a finite impulse response (FIR) filter $h(l)$ to approximate the response of $H(f)$. Denote $\mathbf{g}_{1 j}=\left[g_{1 j}(0) \ldots g_{1 j}\left(2 L p_{1}-1\right)\right]$ as the filtered signature waveform by $h(l), \breve{r}(n)$ as the filtered received signal, and $\breve{\mathbf{r}}=$ $[\breve{r}(n L P) \ldots \breve{r}((n+1) L P-1)]^{T}$, we know that in the absence of noise and assuming perfect out-of-rate interference suppression

$$
\breve{\mathbf{r}}=\mathbf{G}_{1} \mathbf{A}_{1} \mathbf{d}_{1}
$$

where $\mathbf{G}_{1}=\left[\mathbf{G}_{11} \ldots \mathbf{G}_{1 K_{1}}\right]$ with

$$
\begin{aligned}
& \mathbf{G}_{1 j}=\left[\begin{array}{lllll}
\mathbf{g}_{1 j}^{2} & \mathbf{g}_{1 j}^{1} & & & \\
& \mathbf{g}_{1 j}^{2} & \ddots & \mathbf{g}_{1 j}^{1} & \\
& & \ddots & \mathbf{g}_{1 j}^{2} & \mathbf{g}_{1 j}^{1}
\end{array}\right]_{L P \times\left(q_{1}+1\right)} \\
& \mathbf{g}_{1 j}^{1}=\left[g_{1 j}(0) \ldots g_{1 j}\left(L p_{1}-1\right)\right]^{T} \\
& \mathbf{g}_{1 j}^{2}=\left[g_{1 j}\left(L p_{1}\right) \ldots g_{1 j}\left(2 L p_{1}-1\right)\right]^{T} \text {. }
\end{aligned}
$$

Since

$$
\breve{\mathbf{R}}_{1}=E\left(\breve{\mathbf{r}} \breve{\mathbf{r}}^{H}\right)=\mathbf{G}_{1} \mathcal{A}_{1} \mathbf{G}_{1}^{H}
$$

it follows that if $\mathbf{G}_{1}$ has full column rank

$$
\mathcal{A}_{1}=\left(\mathbf{G}_{1}^{H} \mathbf{G}_{1}\right)^{-1} \mathbf{G}_{1}^{H} \breve{\mathbf{R}}_{1} \mathbf{G}_{1}\left(\mathbf{G}_{1}^{H} \mathbf{G}_{1}\right)^{-1}
$$

from which the signal magnitude can be obtained from (24) at the bottom of the page, where the notation $\mathbf{Z}\left(\kappa_{1}: \kappa_{2}, \varsigma_{1}: \varsigma_{2}\right)$ represents the submatrix of $\mathbf{Z}$ from the $\kappa_{1}$ th to the $\kappa_{2}$ th row, and from the $\varsigma_{1}$ th to the $\varsigma_{2}$ th column. With the knowledge of $\left|\beta_{1 j}\right|^{2}$, we can construct $\mathcal{A}_{1}$, and subsequently, $\mathbf{R}_{1}$, which, in turn, yields $\mathbf{R}_{2}$. Performing the eigenvalue decomposition

$$
\mathbf{R}_{2}=\left[\begin{array}{ll}
\breve{\mathbf{U}}_{I} & \breve{\mathbf{U}}_{o}
\end{array}\right]\left[\begin{array}{ll}
\breve{\Sigma}_{I} & \\
& \breve{\Sigma}_{o}
\end{array}\right]\left[\begin{array}{ll}
\breve{\mathbf{U}}_{I} & \breve{\mathbf{U}}_{o}
\end{array}\right]^{H}
$$

the $\left(q_{2}+1\right) K_{2}$ column vectors of $\mathbf{U}_{I}$, which correspond to the $\left(q_{2}+1\right) K_{2}$ (largest) eigenvalues in $\breve{\Sigma}_{I}$, give an estimate of $\mathbf{B}_{I}$. Note that the out-of-rate interference cannot be completely removed by filtering, thus the estimates for $\left|\beta_{1 j}\right|^{2}$ 's are biased, as is the estimate for $\mathbf{B}_{I}$.

\section{B. N-CDMA as the Desired System}

Since the N-CDMA system occupies a fraction of the W-CDMA system's bandwidth, extracting the desired N-CDMA system's signal component by filtering will not suppress the W-CDMA (rate 2) system's interference within the N-CDMA band. Thus, the earlier method (for W-CDMA as the desired system) does not yield the desired estimation accuracy as for N-CDMA's signal magnitudes in this case. However, we can use the multirate signal structure to achieve the purpose.

Denote $\mathbf{r}_{1}=\mathbf{S}_{1} \mathbf{A}_{1} \mathbf{d}_{1}$ and $\mathbf{r}_{2}=\mathbf{S}_{2} \mathbf{A}_{2} \mathbf{d}_{2}$ as the received signals due to the desired N-CDMA system and out-of-rate interfering W-CDMA system, respectively, $\mathbf{r}_{2}^{\varsigma}(\varsigma=1,2)$ as the subvector of $\mathbf{r}_{2}$ consisting of the $\left((\varsigma-1) p_{2} L+1\right)$ th to the $\left(\left(p_{1}+\varsigma-2\right) p_{2} L\right)$ th elements. We can easily verify that

$$
\mathbf{R}_{2}^{1}=E\left(\mathbf{r}_{2}^{1} \mathbf{r}_{2}^{1 H}\right)=E\left(\mathbf{r}_{2}^{2} \mathbf{r}_{2}^{2 H}\right)=\mathbf{R}_{2}^{2} .
$$

With similar definitions for $\mathbf{R}^{\varsigma}$ and $\mathbf{R}_{1}^{\varsigma}(\varsigma=1,2)$, we conclude that

$$
\tilde{\mathbf{R}}=\mathbf{R}^{1}-\mathbf{R}^{2}=\mathbf{R}_{1}^{1}-\mathbf{R}_{1}^{2}=\tilde{\mathbf{R}}_{1} .
$$

Denote $\mathbf{S}_{1}^{\varsigma}(\varsigma=1,2)$ the submatrix of $\mathbf{S}_{1}$ from the $\left((\varsigma-1) p_{2} L+\right.$ $1)$ th to the $\left(\left(p_{1}+\varsigma-2\right) p_{2} L\right)$ th row with possible all-zero columns removed, and $\mathbf{A}_{1}^{\varsigma}$ the corresponding amplitude matrix. We can further rewrite $\tilde{\mathbf{R}}_{1}$ (and hence, $\tilde{\mathbf{R}}$ ) as

$$
\tilde{\mathbf{R}}_{1}=\tilde{\mathbf{S}}_{1} \underbrace{\left[\begin{array}{ll}
\mathbf{A}_{1}^{1} & \\
& -\mathbf{A}_{1}^{2}
\end{array}\right]}_{\Lambda_{1}} \tilde{\mathbf{S}}_{1}^{H}
$$

$$
\left|\beta_{1 j}\right|^{2}=\frac{\operatorname{Trace}\left(\mathcal{A}_{1}\left((j-1)\left(q_{1}+1\right)+1: j\left(q_{1}+1\right),(j-1)\left(q_{1}+1\right)+1: j\left(q_{1}+1\right)\right)\right)}{q_{1}+1}
$$


where $\tilde{\mathbf{S}}_{1}=\left[\mathbf{S}_{1}^{1} \mathbf{S}_{1}^{2}\right]$. Assume $\tilde{\mathbf{S}}_{1}$ has full column rank, then

$$
\Lambda_{1}=\left(\tilde{\mathbf{S}}_{1}^{H} \tilde{\mathbf{S}}_{1}\right)^{-1} \tilde{\mathbf{S}}_{1}^{H} \tilde{\mathbf{R}}_{1} \tilde{\mathbf{S}}_{1}\left(\tilde{\mathbf{S}}_{1}^{H} \tilde{\mathbf{S}}_{1}\right)^{-1}
$$

from which the signal magnitude $\left\|\beta_{1 j}\right\|^{2}$ can be obtained by averaging the diagonal elements of $\Lambda_{1}$ due to user $(1 j)$. With this result and the a priori knowledge of $\mathbf{S}_{1}$, we can estimate $\mathbf{R}_{1}$, which further yields the estimate of $\mathbf{R}_{2}$ by means of subtraction. Similar to (25), we can conduct eigenvalue decomposition on $\mathbf{R}_{2}$, and the $\left(q_{2}+1\right) K_{2}$ eigenvectors corresponding to the largest eigenvalues consist of an estimate of $\mathbf{B}_{I}$. Here we propose another method which saves numerical computations by taking advantage of the multirate signal structure.

It is easy to verify that

$$
\begin{aligned}
\Re_{2}^{\kappa_{1}} & =\mathbf{R}_{2}\left(\left(\kappa_{1}-1\right) L p_{2}+1: \kappa_{1} L p_{2}\left(\kappa_{1}-1\right) L p_{2}+1: \kappa_{1} L p_{2}\right) \\
& =\mathbf{R}_{2}\left(\left(\kappa_{2}-1\right) L p_{2}+1: \kappa_{2} L p_{2}\left(\kappa_{2}-1\right) L p_{2}+1: \kappa_{2} L p_{2}\right) \\
& =\Re_{2}^{\kappa_{2}} \quad \text { for } 1 \leq \kappa_{1}, \quad \kappa_{2} \leq q_{2}
\end{aligned}
$$

since they are all autocorrelation matrices of symbol-length W-CDMA signal snapshots. An eigenvalue decomposition on any of them yields

$$
\Re_{2}^{\kappa}=\left[\begin{array}{ll}
\tilde{\mathbf{U}}_{I} & \tilde{\mathbf{U}}_{o}
\end{array}\right]\left[\begin{array}{cc}
\tilde{\Sigma}_{I} & \tilde{\Sigma}_{o}
\end{array}\right]\left[\begin{array}{ll}
\tilde{\mathbf{U}}_{I} & \tilde{\mathbf{U}}_{o}
\end{array}\right]^{H} .
$$

All $\mathbf{r}_{2}\left((\kappa-1) L p_{2}+1: \kappa L p_{2},(\kappa-1) L p_{2}+1: \kappa L p_{2}\right)$ 's $\left(1 \leq \kappa \leq q_{2}\right)$ lie in the $2 K_{2}$-dimensional signal subspace $\tilde{\mathbf{U}}_{I}$, indicating

$$
\mathbf{r}_{2}=\underbrace{\left[\begin{array}{ccc}
\tilde{\mathbf{U}}_{I} & & \\
& \ddots & \\
& & \tilde{\mathbf{U}}_{I}
\end{array}\right]}_{\mathbf{B}_{I}: q_{2} \text { blocks }}\left[\begin{array}{ccc}
\tilde{\mathbf{U}}_{I} & & \\
& \ddots & \\
& & \tilde{\mathbf{U}}_{I}
\end{array}\right]^{H} \mathbf{r}_{2} .
$$

Thus $\mathbf{B}_{I}$ can also be constructed as above. In practice, we recommend using $\Re_{2}=\sum_{\kappa=1}^{q_{2}} \Re_{2}^{\kappa}$ for the calculation of $\tilde{\mathbf{U}}_{I}$ to improve noise immunity.

\section{Estimation of the Number of Out-of-Rate Fictitious Interferers}

In the previous sections, we have assumed that the number of out-of-rate interferers is known. In practice, heterogeneous systems usually do not exchange such information, and therefore, the number has to be estimated. It is seen that the Akaike information criterion (AIC) [19] can be used upon the estimate of $\mathbf{R}_{2}$ or $\Re_{2}$ to achieve this purpose.

\section{Complexity}

The major computational demands of the proposed methods come from the matrix inversion for signal magnitudes estimation and the eigenvalue decomposition for out-of-rate interference subspace estimation. For the W-CDMA system, the inversion is conducted on a matrix of dimension $\left(q_{1}+1\right) K_{1} \times\left(q_{1}+1\right) K_{1}$, which incurs $(8 / 3)\left(q_{1}+1\right)^{3} K_{1}^{3}+\mathcal{O}\left(\left(q_{1}+1\right)^{2} K_{1}^{2}\right)$ flops; the eigenvalue decomposition is conducted on a matrix of dimension $L P \times L P$, which incurs $\mathcal{O}\left(L^{3} P^{3}\right)$ flops. For the N-CDMA system, the inversion is conducted on a matrix of dimension $2 q_{1} K_{1} \times 2 q_{1} K_{1}$, which incurs $(64 / 3) q_{1}^{3} K_{1}^{3}+\mathcal{O}\left(4 q_{1}^{2} K_{1}^{2}\right)$ flops; the eigenvalue decomposition is conducted on a matrix of dimension $L \times L$ which incurs $\mathcal{O}\left(L^{3}\right)$ flops. In comparison with the method proposed in [14], the same order of complexity is involved for the W-CDMA system, due to the inversion and eigenvalue decomposition of matrices of exactly the same dimensions. Fewer computations are required for the N-CDMA system in the proposed algorithm, since the method in [14] treats W-CDMA and N-CDMA systems the same way.

\section{E. Spreading Codes Assignment}

To use the PIC structure, we focus on the estimation of interfering subspace $\mathbf{B}_{I}$. Toward that end, we only require $\mathbf{G}_{1}$ and $\tilde{\mathbf{S}}_{1}$ to be of full rank, as seen in (23) and (28), which can be satisfied since the two matrices are determined by the spreading codes of the same system. However, it is well known that for the equivalent single-rate signal model (9), detection performance is severely degraded if $\mathbf{S}$ loses the full-rank property. This can be a concern if each system allocates its spreading codes independently. Luckily, when a system is turned on, the chance of many users being admitted simultaneously is very low. We, therefore, assume $\mathbf{S}$ is of full rank at some time instant. When a new user requests to enter the system, the base takes advantage of the recent $\mathbf{B}_{I}$ estimate. A spreading code which is linearly independent of, ideally of low cross-correlation with, both $\mathbf{S}_{1}$ and $\mathbf{B}_{I}$, is assigned to guarantee the full-rank property of $\mathbf{S}$. If such a code does not exist, the user is refused admission, a price that has to be paid to facilitate mitigation from a legacy narrowband system to a new wideband system, although the same problem is also seen in a single-rate system.

\section{F. Long Versus Short Spreading Codes}

During the algorithm development, we have assumed short spreading codes for both W-CDMA and N-CDMA systems. It is seen that our method still applies as long as the newer W-CDMA system adopts short spreading codes, which has gained more and more acceptance now because it facilitates MUD for performance improvement. However, it does not seem to be easy to generalize the approaches to situations where all systems use long spreading codes.

\section{MEAN OUTPUT SINR}

Following [15], we analyze the asymptotic performance of the proposed methods in terms of mean output signal-to-interference-plus-noise ratio (SINR) when the signature waveforms are fixed. We denote $\Delta \mathbf{Z}(\Delta \mathbf{Z})$ the perturbation to the variable $\mathbf{z}(\mathbf{Z}), k$ the index of the desired fictitious user within the vector $\mathbf{d}_{1}, \mathbf{r}_{1 k}$ its contribution to the received signal $\mathbf{r}$, and $\mathbf{f}_{k}$ the $k$ th column of $\mathbf{F}_{M}$ which is the corresponding receive filter. We see that the signal component's amplitude is $\beta_{1 k^{\prime}}$, where $k^{\prime}=\left\lceil k / q_{1}\right\rceil$, and $\mathbf{S}_{1 k}$, the $k$ th column of $\mathbf{S}_{1}$, is the associated signature waveform. Given $\mathbf{f}_{k}$, the output SINR for the fictitious user is defined as [12]

$$
\operatorname{SINR}_{k}=\frac{\left|\beta_{1 k^{\prime}}\right|^{2} \mathbf{f}_{k}^{H} \mathbf{S}_{1 k} \mathbf{S}_{1 k}^{H} \mathbf{f}_{k}}{\mathbf{f}_{k}^{H}\left(\sigma^{2} \mathbf{I}+\check{\mathbf{S}}_{1 k} \check{\mathbf{A}}_{1 k} \check{\mathbf{A}}_{1 k}^{H} \check{\mathbf{S}}_{1 k}^{H}\right) \mathbf{f}_{k}}=\frac{\mathbf{f}_{k}^{H} \mathbf{X} \mathbf{f}_{k}}{\mathbf{f}_{k}^{H} \mathbf{Y} \mathbf{f}_{k}}
$$

where $\breve{\mathbf{S}}_{1 k}$ and $\check{\mathbf{A}}_{1 k}$ are, respectively, obtained by removing $\mathbf{S}_{1 k}$ and its amplitude from $\mathbf{S}$ and $\mathbf{A}, \mathbf{X}, \mathbf{Y}$ are defined implicitly. For a perturbed version of the receive filter $\hat{\mathbf{f}}_{k}=\mathbf{f}_{k}+\Delta \mathbf{f}_{k}$, an 
approximation to the $\mathrm{SINR}_{k}$ estimate follows using the firstorder Taylor formula [18]:

$$
\begin{array}{r}
\widehat{\mathrm{SINR}}_{k} \approx \mathrm{SINR}_{k}+\left(\left(\mathbf{f}_{k}^{H} \mathbf{Y} \mathbf{f}_{k}\right)\left(\Delta \mathbf{f}_{k}^{H} \mathbf{X} \mathbf{f}_{k}+\mathbf{f}_{k}^{H} \mathbf{X} \Delta \mathbf{f}_{k}\right)\right. \\
\left.-\left(\mathbf{f}_{k}^{H} \mathbf{X} \mathbf{f}_{k}\right)\left(\Delta \mathbf{f}_{k}^{H} \mathbf{Y} \mathbf{f}_{k}+\mathbf{f}_{k}^{H} \mathbf{Y} \Delta \mathbf{f}_{k}\right)\right) /\left(\mathbf{f}_{k}^{H} \mathbf{Y} \mathbf{f}_{k}\right)^{2} .
\end{array}
$$

The mean of the output SINR up to the first order is given by

$$
\begin{aligned}
E\left(\widehat{S I N R}_{k}\right) \\
\approx \operatorname{SINR}_{k}+\left(\left(\mathbf{f}_{k}^{H} \mathbf{Y} \mathbf{f}_{k}\right)\left({\overline{\Delta \mathbf{f}_{k}}}^{H} \mathbf{X} \mathbf{f}_{k}+\mathbf{f}_{k}^{H} \mathbf{X} \overline{\Delta \mathbf{f}_{k}}\right)\right. \\
\left.\quad-\left(\mathbf{f}_{k}^{H} \mathbf{X} \mathbf{f}_{k}\right)\left({\overline{\Delta \mathbf{f}_{k}}}^{H} \mathbf{Y} \mathbf{f}_{k}+\mathbf{f}_{k}^{H} \mathbf{Y} \overline{\Delta \mathbf{f}_{k}}\right)\right) /\left(\mathbf{f}_{k}^{H} \mathbf{Y} \mathbf{f}_{k}\right)^{2}
\end{aligned}
$$

where overbar on a variable indicates the mean value. Since the first-order perturbation to $\mathbf{F}_{M}$ is a linear function of $\Delta \mathcal{S}, \overline{\Delta \mathbf{F}_{M}}$ (and hence, $\overline{\Delta \mathbf{f}_{k}}$ ) can be approximated with $\overline{\Delta \mathcal{S}}$ by expanding (17) and only keeping the first-order terms. Note that $\overline{\Delta \mathcal{S}}=$ $\left[\mathbf{0} \overline{\Delta \mathbf{B}_{I}}\right]$ due to exact knowledge of $\mathbf{S}_{1}$. In the following, we derive the estimate of $\overline{\Delta \mathbf{B}_{I}}$ when the desired system is, respectively, W-CDMA and N-CDMA.

\section{A. W-CDMA as the Desired System}

Considering the residual out-of-rate interference and additive noise, the perturbation to $\breve{\mathbf{r}}$ is given by

$$
\Delta \breve{\mathbf{r}}=\mathbf{G}_{2} \mathbf{A}_{2} \mathbf{d}_{2}+\mathbf{G}_{w} \breve{\mathbf{w}} .
$$

Denote by $\mathbf{g}_{2 j}$ the filtered signature waveforms of the out-ofrate interferers. $\mathbf{G}_{2}$ is constructed from $\mathbf{g}_{2 j}$ in the same way as $\mathbf{G}_{1}$ from $\mathbf{g}_{1 j} \cdot \mathbf{G}_{w}$ and $\breve{\mathbf{w}}$ are, respectively, given as

$$
\begin{aligned}
& \mathbf{G}_{w}=\underbrace{\left[\begin{array}{ccccc}
h(N) & \cdots & h(0) & & \\
& \ddots & & \ddots & \\
& & h(N) & & h(0)
\end{array}\right]}_{L P \times(L P+N)} \\
& \breve{\mathbf{w}}=\left[w(n L P \delta-N) \delta \ldots w((n L P+L P-1) \delta]^{T}\right. \text {. }
\end{aligned}
$$

Thus we know that $\Delta \breve{\mathbf{R}}_{1}=\mathbf{G}_{2} \mathcal{A}_{2} \mathbf{G}_{2}^{H}+\sigma^{2} \mathbf{G}_{w} \mathbf{G}_{w}^{H}$, from which we can obtain $\Delta \breve{\mathcal{A}}_{1}, \Delta\left|\beta_{1 j}\right|^{2}$ from (23) and (24), respectively, and hence, $\Delta \mathbf{R}_{2}$. It is shown in [17] that up to first-order

$$
\Delta \mathbf{B}_{I} \approx \breve{\mathbf{U}}_{o} \breve{\mathbf{U}}_{o}^{H} \Delta \mathbf{R}_{2} \mathbf{B}_{I} \breve{\Sigma}_{I}^{-1}
$$

where $\breve{\mathbf{U}}_{O}$ is the subspace of $\breve{\mathbf{U}}$, which consists of $\mathbf{B}_{I}$ 's orthogonal complement, and $\breve{\Sigma}_{I}$ is the diagonal matrix of eigenvalues corresponding to the vectors in $\mathbf{B}_{I}$. It is easy to see that $\overline{\Delta \mathbf{B}_{I}}=\Delta \mathbf{B}_{I}$.

\section{B. N-CDMA as the Desired System}

Note from (26)-(28) that the estimates for $\left\|\beta_{1 j}\right\|^{2}$ 's are unbiased. Thus the perturbation to $\mathbf{R}_{2}$, and hence, $\Re_{2}$, is only due to additive noise, i.e., $\Delta \Re_{2}=q_{2} \sigma^{2} \mathbf{I}$. From [17], we obtain up to the first order that

$$
\Delta \tilde{\mathbf{U}}_{I} \approx \tilde{\mathbf{U}}_{o} \tilde{\mathbf{U}}_{o}^{H} \Delta \Re_{2} \tilde{\mathbf{U}}_{I} \tilde{\Sigma}_{I}^{-1} .
$$

$\overline{\Delta \mathbf{B}_{I}}$ can then be determined from $\mathbf{B}_{I}$ 's definition in (31), accordingly.

\section{Simulation Results}

Various aspects of the proposed methods are investigated in this section. For comparison, we simulate: 1) the conventional MMSE receiver where only the desired user's signature waveform is needed; 2) the form I group-blind receiver [14] where the desired system's known signature waveforms are all used to extract the out-of-rate interference component perpendicular to them, which is then exploited to construct the detector; and 3) conventional PIC, after filtering the received signal with either (20) for the W-CDMA system, or with

$$
H(f)=\frac{\mathcal{Z}_{N}(f)}{\mathcal{Z}_{W}(f)+\mathcal{Z}_{N}(f)}
$$

for the N-CDMA system. Note that in this case, the effective signature waveforms, i.e., the convolution of known signature waveforms with the filter response, are used. The order of the modified Wiener filter is chosen such that no more performance gain can be obtained. We consider dual-rate systems with the rectangular chip pulse-shaping function. Random binary short spreading codes with equiprobable \pm 1 elements are used, which satisfy the convergence condition in [11]. Note that the spreading codes are generated at the beginning of the simulation, and fixed afterwards for all trials. Uncoded binary phase-shift keying (BPSK) is used to modulate the data symbols. We normalize the carrier offset with respect to the W-CDMA system's bandwidth. 600 data sample vectors are exploited to estimate $\mathbf{B}_{I}$, and 100 independent trials are conducted to compute the mean of the performance index of interest. Without loss of generality, we look at the performance of user 1 in the desired system.

Example 1: We test an overlay application where rate ratio is $1: 3$, carrier offset is 0.2 , processing gain is 16 for both systems, and there are two users in each of them. The same symbol energy $E_{s}$ is used in both systems, and three-stage receivers are exploited. The performance curves versus signal-to-noise ratio $\mathrm{SNR}=E_{s} / \sigma^{2}$ are depicted in Figs. 4 and 5 . The results of matched filtering are also shown. It is seen that for both N-CDMA and W-CDMA systems, the proposed PIC methods clearly outperform all other algorithms. Further, the group-blind form-I detector is better than the MMSE receiver, as reported in [14]. The suppression-filtering-plus-PIC scheme behaves differently in the W-CDMA and N-CDMA systems. For the W-CDMA system, suppression filtering helps to collect the portion of W-CDMA signal that does not overlap the $\mathrm{N}-\mathrm{CDMA}$ signal in spectrum. Therefore, out-of-rate interference is effectively removed. Our simulations indicate that such a receiver can achieve performance comparable to that of the group-blind form I detector [14] at high SNR, while providing close to matched-filtering performance at low SNRs. This is due to the fact that the noise power is not considered in the modified filter in comparison with the optimal Wiener filter. For the N-CDMA system, it is impossible to effectively remove the out-of-rate W-CDMA interference by filtering; therefore, PIC is conducted with only some, instead of all, of the effective signature waveforms that define the filtered signal. Besides, filtering introduces interblock interference. All these factors 


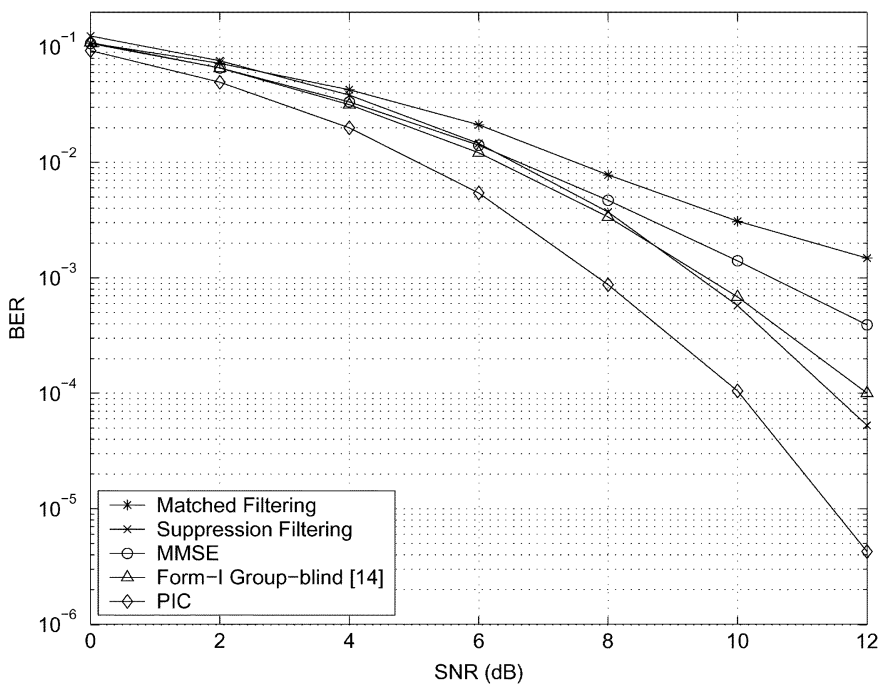

Fig. 4. BER of the W-CDMA system as a function of SNR. $L=16, \Delta f_{2}=$ 0.2 , rate ratio $1: 3$, two N-CDMA/W-CDMA users, $\mathrm{SIR}=0 \mathrm{~dB}$

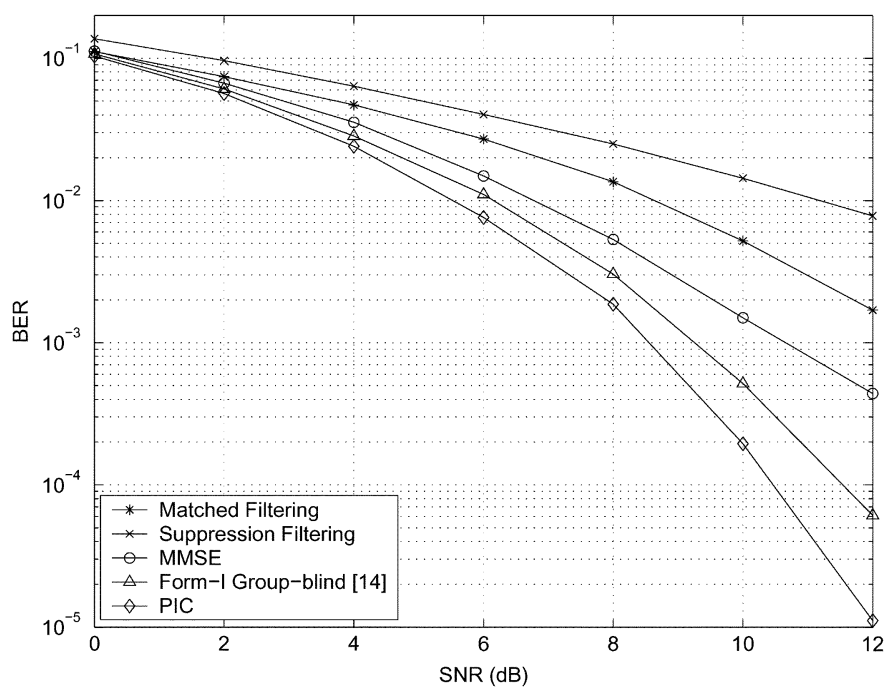

Fig. 5. BER of the N-CDMA system as a function of SNR. $L=16, \Delta f_{2}=$ 0.2 , rate ratio $1: 3$, two $\mathrm{N}$-CDMA/W-CDMA users, $\mathrm{SIR}=0 \mathrm{~dB}$.

lead to poorer results than even matched filtering. In summary, we attribute the superior performance of the proposed methods to a combination of: a) the group-blind MUD architecture that better exploits the available information and depends less on estimated parameters in comparison with the MMSE detector and suppression filtering; and b) improved estimation of the number of out-of-rate interferers and their associated subspace, in comparison with the form-I group-blind detector for reasons already described.

Example 2: Since there is no coordinated power control between overlay systems, the algorithms' capacity to suppress out-of-rate interference is of critical importance. Denoting $E_{1}$ the symbol energy shared by all the users in the desired system, and $E_{2}$ the symbol energy for all the users in the interfering system, we test the methods across a wide range of signal to (out-of-rate) interference ratio SIR $=E_{1} / E_{2}$. With the dual-rate system configured as in Example 1, except SNR $=8$ $\mathrm{dB}$ and varying SIR values, we obtain results shown in Fig. 6.

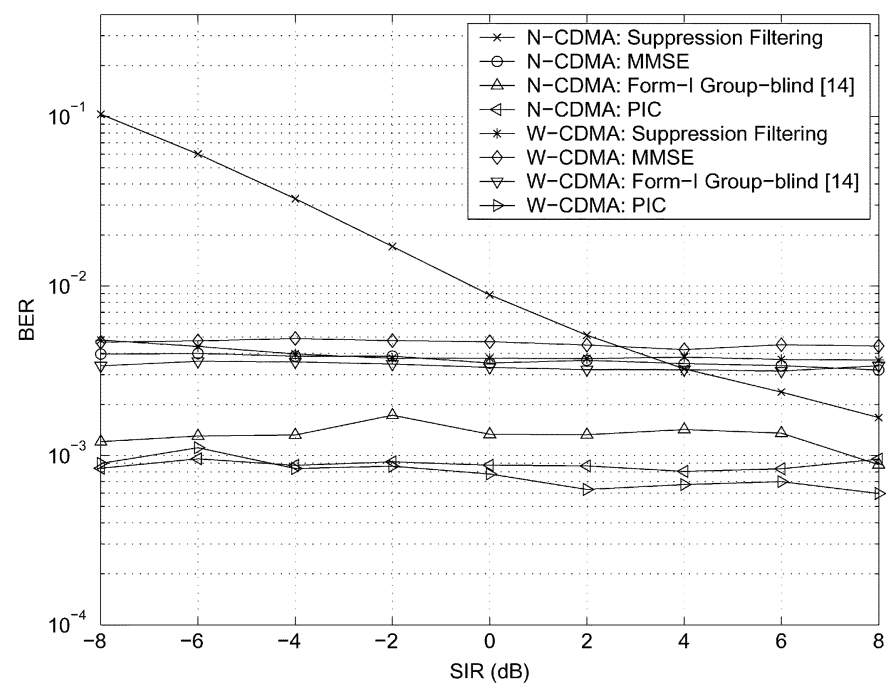

Fig. 6. BER as a function of SIR. $L=16, \Delta f_{2}=0.2$, rate ratio 1:3, two $\mathrm{N}$-CDMA/W-CDMA users, $\mathrm{SNR}=8 \mathrm{~dB}$.

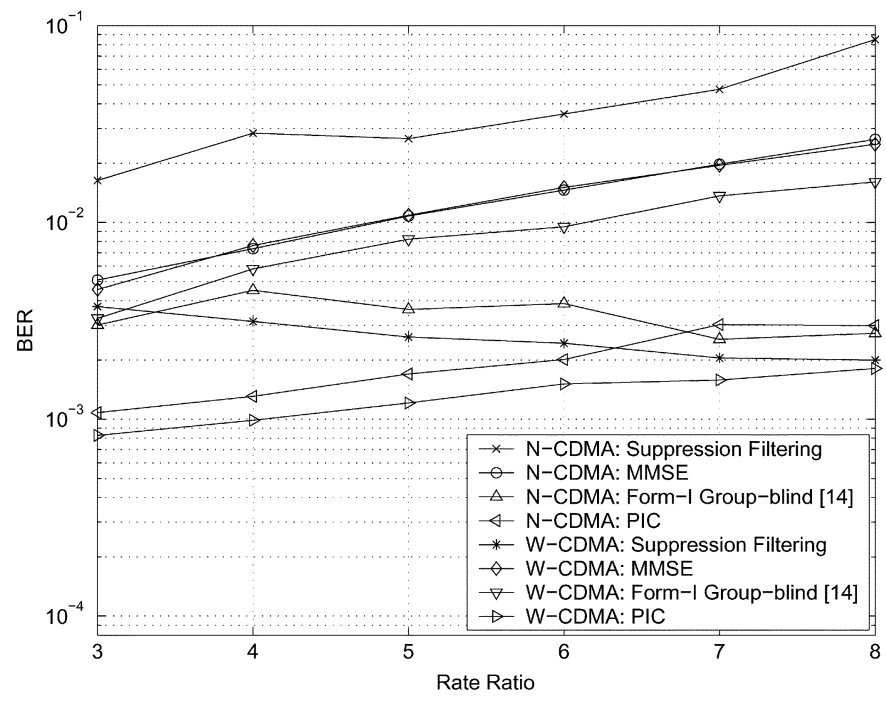

Fig. 7. BER as a function of rate ratio. $L=16, \Delta f_{2}=0.2$, two N-CDMA/W-CDMA users, $\operatorname{SIR}=0 \mathrm{~dB}, \mathrm{SNR}=8 \mathrm{~dB}$.

We see that all the receivers are immune to SIR variation except the suppression-filtering-plus-PIC solution for the N-CDMA system, which is true because this is not really a multiuser detector, as explained in Example 1.

Example 3: An important issue in overlay communications is how the rate ratio affects the constituent systems' performance. We use the system specifications of Example 1, except that the rate ratio is variable and $\mathrm{SNR}=8 \mathrm{~dB}$. The simulation results are depicted in Fig. 7. It is seen that as the rate ratio increases, the performance of the conventional MMSE receiver degrades. This is because the dimension of the receive filter increases accordingly, and thus, leads to poorer receiver estimates. Given the same symbol energy, as the rate ratio increases, the W-CDMA signal becomes relatively stronger in comparison with the N-CDMA signal, which means a) stronger signal-tonoise power ratio for the W-CDMA system when the N-CDMA system is the desired system, and b) lower signal-to-noise power ratio for the N-CDMA system when the W-CDMA system is the 


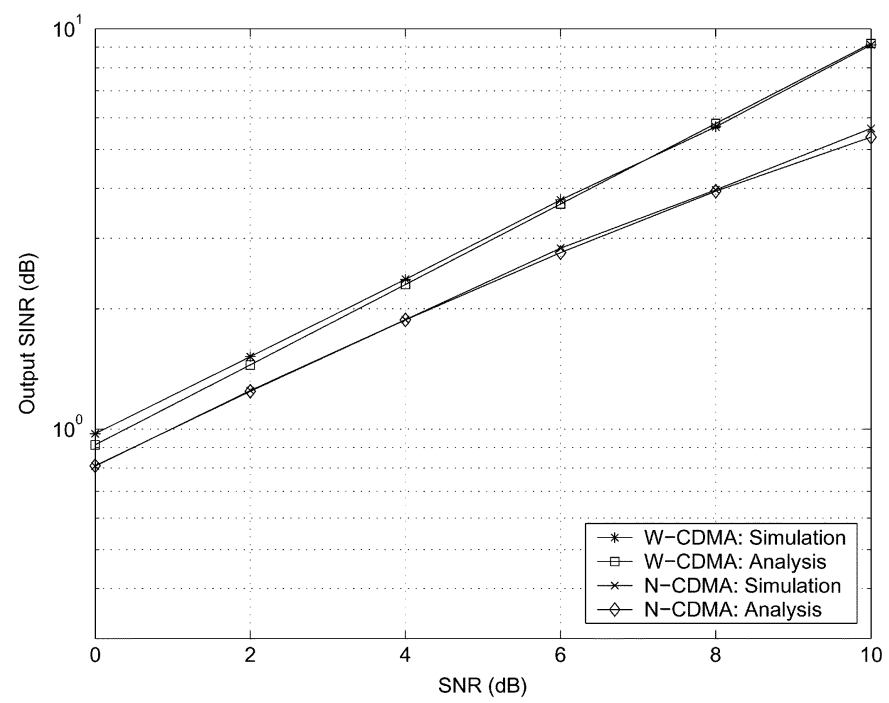

Fig. 8. Mean output SINR. $L=16, \Delta f_{2}=0.2$, rate ratio $1: 3$, two $\mathrm{N}$-CDMA/W-CDMA users, SIR $=10 \mathrm{~dB}$.

desired system. Therefore, the larger the rate ratio, the higher the level of out-of-rate interference experienced by the desired $\mathrm{N}$-CDMA system, and hence, the poorer is the performance of the suppression-filtering approach. On the other hand, as the rate ratio becomes larger, the W-CDMA system has more spectrum that is out-of-rate-interference-free, which explains why, in contrast to the N-CDMA system, suppression filtering generates better performance. For the group-blind form-I detectors, we observe two opposite trends when the desired system is, respectively, W-CDMA or N-CDMA. Due to reason b) discussed above, the out-of-rate interference signal used for estimation becomes relatively weaker against noise when the W-CDMA signal is desired and the rate ratio increases, which leads to poorer performance. Similarly, we can reason from a) why performance is improved when the N-CDMA signal is detected. For the proposed methods, as the rate ratio increases, an autocorrelation matrix of larger dimension needs to be inverted. This results in poorer estimates of signal magnitudes, and hence $\mathbf{B}_{I}$, translating into performance degradation. Our simulation results support the performance advantages of the new PIC detectors.

Example 4: We now test the analytical results in Section IV against simulations with the overlay scenario specified in $E x$ ample 1. The curves are depicted in Fig. 8. Clearly, we can see that the analysis matches simulation results very well. It is seen that W-CDMA has better performance than N-CDMA, which agrees with the BER curves through previous examples. This is due to the fact that the W-CDMA system has a larger power level, therefore, its interference to the N-CDMA system is stronger than what it experiences from the N-CDMA system.

Example 5: Now we show the performance improvements when more interference-cancellation stages are added. For an overlay of processing gain 24 for both W-CDMA and N-CDMA systems, five users in each, rate ratio $1: 4$, carrier offset 0.15 , and $\mathrm{SIR}=0 \mathrm{~dB}$, we obtain the results shown in Fig. 9. It is seen that as the number of stages increases, the proposed detectors converge quickly. Also, performance of the N-CDMA system improves faster than that of the W-CDMA system as SNR becomes larger. This is because for the W-CDMA system, the filtering

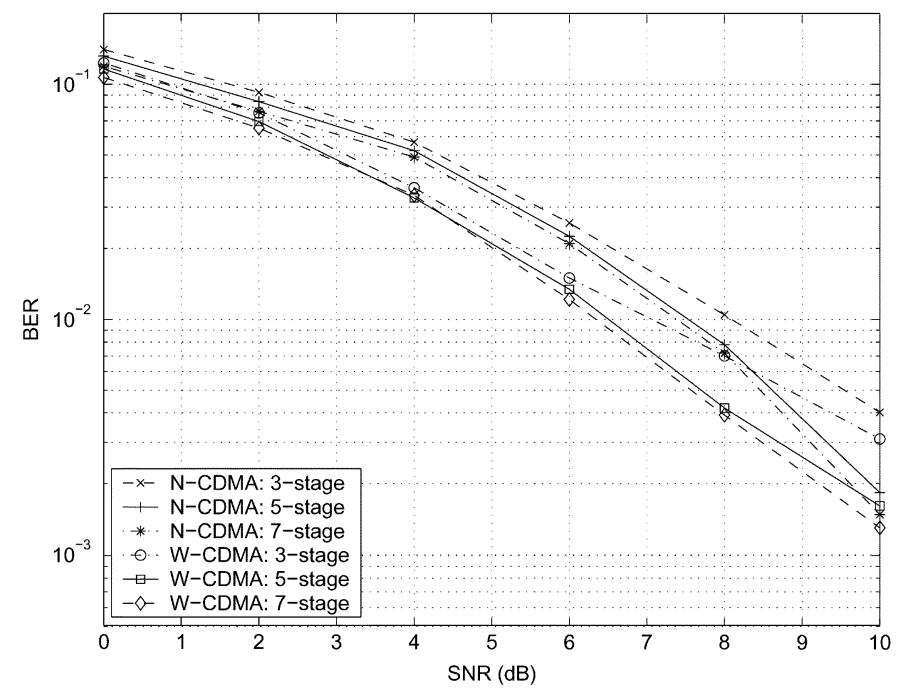

Fig. 9. BER as a function of stage number. $L=24, \Delta f_{2}=0.15$, rate ratio $1: 4$, five N-CDMA/W-CDMA users, SIR $=0 \mathrm{~dB}$.

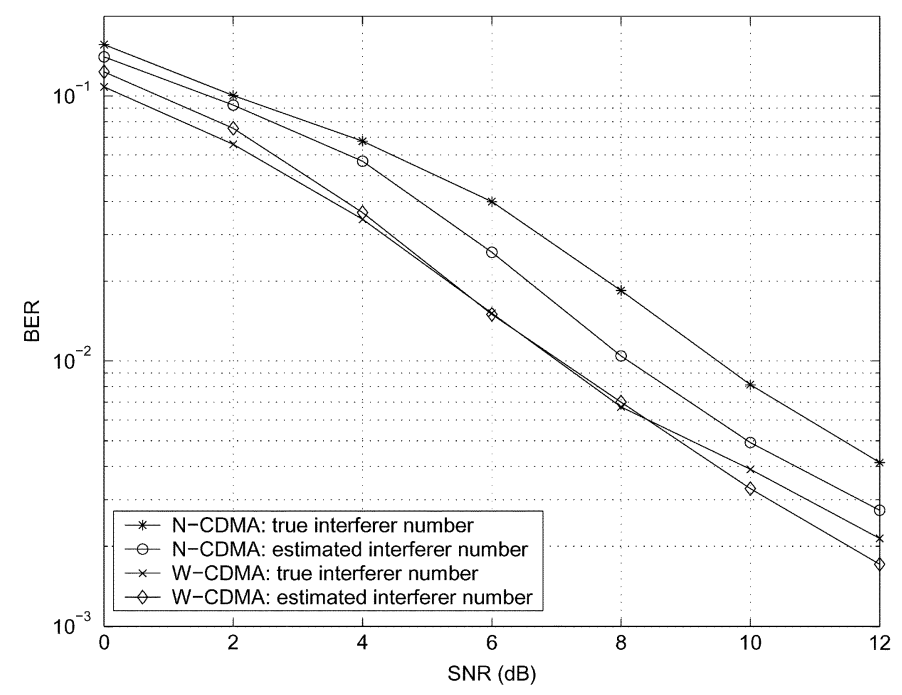

Fig. 10. Impact of imperfect knowledge of the number of out-of-rate interferers. $L=24, \Delta f_{2}=0.15$, rate ratio $1: 4$, five N-CDMA/W-CDMA users, SIR $=0 \mathrm{~dB}$.

process always incurs a bias in the estimates of signal magnitudes. In contrast, for the N-CDMA system, the estimates are unbiased, and thus, approach the true values when noise level is lower.

Example 6: Finally, we study how the estimate of the number of out-of-rate interferers affects the receiver performance. The overlay described in Example 5 is simulated with the three-stage receivers. In Fig. 10, results for both perfect and imperfect knowledge of this number are illustrated. We see that for the W-CDMA system imperfect estimates of the number does not have an obvious effect on the performance; for the N-CDMA system, imperfect estimates actually generates better performance. Further investigation indicates that accurate estimates are usually obtained for the W-CDMA system, while underestimation appears to be more typical for the N-CDMA system, which happens when out-of-rate interference components along the directions of some eigenvectors are close to noise in power. In such cases, those eigenvectors 
will generate unreliable estimates if included in $\mathbf{B}_{I}$. Therefore, better performance is expected when only the eigenvectors that collect most, but not all, of the out-of-rate interference are used.

\section{CONCLUSION}

A group-blind MUD scheme is proposed which deals with overlay applications involving multiple CDMA systems. A multirate communications framework is used to develop a PIC receiver structure whose effectiveness is demonstrated by computer simulations. Throughout the paper, we have assumed static frequency-nonselective channels. For wideband frequency-selective channels, sophisticated channel-estimation techniques [13] will be needed to construct the effective signature waveforms to replace the spreading codes. Some detailed investigations, such as suppression-filter selection for the W-CDMA system, may also benefit system performance. In the paper, we have chosen the modified Wiener filter for the simplicity of receiver design. As far as the detection performance is concerned, the filter should be designed for accurate estimates of signal magnitudes $\left\|\beta_{1 j}\right\|$ 's, which indicates the necessity to balance the detrimental effects of both noise and out-of-rate N-CDMA interference.

\section{REFERENCES}

[1] L. Milstein et al., "On the feasibility of a CDMA overlay for personal communications networks," IEEE J. Select. Areas Commun., vol. 10, pp. 655-667, May 1992.

[2] R. L. Pickholtz, L. B. Milstein, and D. L. Schilling, "Spread spectrum for mobile communications," IEEE Trans. Veh. Technol., vol. 40, pp. 313-322, May 1991.

[3] P. Koorevaar and J. Ruprecht, "Frequency overlay of GSM and cellular B-CDMA," IEEE Trans. Veh. Technol., vol. 48, pp. 696-707, May 1999.

[4] L. Noel and T. Widdowson, "Experimental CDMA data overlay of GSM network," Electron. Lett., vol. 35, no. 8, pp. 614-615, Apr. 1999.

[5] T. Widdowson and L. Noel, "Uplink and downlink experimental CDMA overlay of GSM network in fading environment," Electron. Lett., vol. 35, no. 17, pp. 1440-1441, Aug. 1999.

[6] L. A. Rusch and H. Poor, "Multiuser detection techniques for narrowband interference suppression in spread spectrum communications," IEEE Trans. Commun., vol. 43, pp. 1725-1737, Feb.-Apr. 1995.

[7] H. Poor, "Active interference cancellation in CDMA overlay systems," IEEE J. Select. Areas Commun., vol. 19, pp. 4-20, Jan. 2001.

[8] M. K. Varanasi and B. Aazhang, "Multistage detection in asynchronous code-division multiple-access communications," IEEE Trans. Commun., vol. 38, pp. 509-519, Apr. 1990.

[9] D. Guo, L. Rasmussen, S. Sun, and T. Lim, "A matrix-algebraic approach to linear parallel interference cancellation in CDMA," IEEE Trans. Commun., vol. 48, pp. 152-161, Jan. 2000.

[10] A. Kaul and B. Woerner, "Analytic limits on the performance of adaptive multistage interference cancellation," Electron. Lett., vol. 30, pp. 2093-2094, Dec. 1994.

[11] V. Ghazi-Moghadam, L. Nelson, and M. Kaveh, "Parallel interference cancellation for CDMA systems," in Proc. 33rd Annu. Allerton Conf. Communications, Control, Computing, Monticello, IL, Oct. 1995, pp. 216-224.
[12] U. Madhow and M. L. Honig, "MMSE interference suppression for direct-sequence spread-spectrum CDMA," IEEE Trans. Commun., vol. 42, pp. 3178-3188, Dec. 1994.

[13] S. Roy and H. Yan, "Blind channel estimation in multirate CDMA systems," IEEE Trans. Commun., vol. 50, pp. 995-1004, June 2002.

[14] X. Wang and H. V. Poor, "Group-blind multiuser detection for uplink CDMA," IEEE J. Select. Areas Commun., vol. 17, pp. 1971-1984, Nov. 1999.

[15] A. Host-Madsen and X. Wang, "Performance of blind and group-blind multiuser detection," IEEE Trans. Inform. Theory, vol. 48, pp. 1849-1872, Jul. 2002.

[16] T. Moon, Z. Xie, C. Rushforth, and R. Short, "Parameter estimation in a multiuser communication system," IEEE Trans. Commun., vol. 42, pp. 2553-2560, Aug. 1994.

[17] F. Li and R. Vaccaro, "Unified analysis for DOA estimation algorithms in array signal processing," Signal Processing, vol. 25, pp. 147-169, Nov. 1991.

[18] S. Malik, Mathematical Analysis. New Delhi, India: Halsted, 1984.

[19] M. Wax and T. Kailath, "Detection of signals by information theoretic criteria," IEEE Trans. Acoust. Speech, Signal Processing, vol. ASSP-33, pp. 387-392, Apr. 1985.

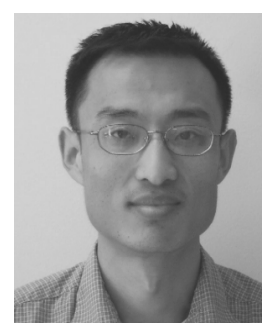

Hongbo Yan (M'02) received the B.S. degree from Northwestern Polytechnic University, Xi'an, China, in 1995, the M.S. degree from Tsinghua University, Beijing, China, in 1998, and the Ph.D. degree from the University of Washington, Seattle, in 2002.

$\mathrm{He}$ is now a Senior System Engineer with Qualcomm Inc., San Diego, CA, working on system aspects of $3 \mathrm{G}$ wireless networks. His research interests include various issues on OFDM and CDMA systems and wireless networking.

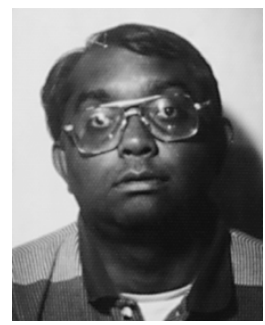

Sumit Roy (S'84-M'88-SM'00) received the B.Tech. degree from the Indian Institute of Technology, Kanpur, India, in 1983, and the M.S. and Ph.D. degrees from the University of California, Santa Barbara (UC Santa Barbara), in 1985 and 1988 , respectively, all in electrical engineering, and the M.A. degree in statistics and applied probability in 1988, also from UC Santa Barbara.

His previous academic appointments were at the Moore School of Electrical Engineering, University of Pennsylvania, and the University of Texas, San Antonio. Currently, he is a Professor of Electrical Engineering with the University of Washington, Seattle, where his research interests include analysis/design of communication systems/networks, with a topical emphasis on next-generation mobile/wireless networks. He spent 2002-2003 on academic leave at the Intel Wireless Technology Laboratory, Seattle, WA, working on system design of next-generation IEEE LANs and PANs (ultra-wideband radios). He currently serves on the Editorial Board for Wiley's Journal of Wireless Communications and Mobile Computing.

Dr. Roy's activities for the IEEE Communications Society include membership of several technical committees and conference program committees. $\mathrm{He}$ recently completed a term as Editor for the IEEE TRANSACTIONS ON WIRELESS COMMUNICATIONS. 